\title{
Reconfigurable Direct Space-to-Time Pulse-Shaper Based on Arrayed Waveguide Grating Multiplexers and Digital Micromirrors
}

\author{
A. Krishnan, M. Knapczyk, L. Grave de Peralta, A. A. Bernussi, and H. Temkin
}

\begin{abstract}
We describe a dynamic direct space-to-time pulse shaper based on the combination of a reflective arrayed waveguide grating multiplexer and an array of digital micromirrors used as an external reflector. Spatial modulation was obtained by imposing a reconfigurable periodic structure on the digital micromirror array. The period of this structure and the number of reflecting micromirrors within each period was varied to produce pulse sequences with variable repetition rates within the burst and different pulsewidths. Experimental results are in excellent agreement with simulated temporal output response.
\end{abstract}

Index Terms-Optical pulse shaping, planar waveguides, waveguide arrays, wavelength-division multiplexing (WDM).

$\mathbf{G}$ ENERATION of bursts of femtosecond pulses with very high repetition rates is critical to the implementation of optical code-division multiple-access [1] and optically assisted internet routing [2] networks. Reconfigurable terahertz bursts of pulses have been demonstrated using programmable bulk optics Fourier transform pulse shapers (PS) with liquid-crystal modulators and acoustic optic modulators as spatial masks [3], [4]. Direct space-to-time (DST) is another approach to the generation of ultrafast optical pulse sequences [5]. More recently, very high repetition rates of ultrashort pulses have been demonstrated using specially designed arrayed waveguide grating (AWG) devices [6]. While the latter approach offers considerable footprint advantage over bulk optics, it does not allow for reconfigurability, limiting its application for pulse encoding. In order to extend the performance of AWG-based pulse shapers for encoding applications, we experimented with a dynamic reflective (R)-AWG based on a combination of an R-AWG and a digital micromirror device (DMD) used as an external reflector [7]. Previously, we investigated the spectral response of the R-AWG-DMD combination and demonstrated that this approach allows for dynamic modification of the free spectral range (FSR) of the R-AWG [7].

In this study, we investigate the temporal response of a reconfigurable DST-PS based on the combination of an R-AWG and an array of digital micromirrors. As expected on the basis of previous experiments [6], [7], modified FSR results in pulse sequences with different repetition rates within the output pulse

Manuscript received March 2, 2005; revised April 7, 2005. This work was supported by Defense Advanced Research Projects Agency (DARPA) and by the Jack F. Maddox Foundation.

The authors are with the Department of Electrical and Computer Engineering, Texas Tech University, Lubbock, TX 79409 USA (e-mail: ayrton.bernussi@ttu.edu).

Digital Object Identifier 10.1109/LPT.2005.852332 train. We also show that the width of the pulses within the train can be modified. These results are quantified and compared, for the first time, with a simulation of the temporal response of the R-AWG. The simulation takes into account the phase and intensity values calculated for each waveguide of the grating. An excellent agreement between the simulated and measured temporal response of the reconfigurable pulse shaper is obtained.

In this work, spatial modulation was obtained by imposing a reconfigurable reflecting structure on the DMD. The period of this structure and the number of reflecting micromirrors within each period was varied to produce pulse sequences with different repetition rates and different pulsewidths. The output response of the R-AWG strongly depends on the number of grating waveguides contributing $\left(N_{\mathrm{wg}}^{\mathrm{ON}}\right)$ and not contributing $\left(N_{\mathrm{wg}}^{\mathrm{OFF}}\right)$ to the device response in each DMD period [7].

Details of the experimental setup and the characteristics of R-AWGs and DMDs used in this work were described previously [7], [8].

In the experiments presented here, a passive mode-locked fiber laser generating 500 -fs pulses at the repetition rate of $50 \mathrm{MHz}$ and the center wavelength of $1560 \mathrm{~nm}$ was used as the input source (Femtomaster laser from Fianium). The temporal characteristics of the R-AWG output channels were obtained using free-space intensity autocorrelation measurements.

Fig. 1 shows the spectra and temporal output profiles of a single output channel of an R-AWG with all micromirrors of the DMD set to the reflecting state (ON-state) or to a period with 14 waveguides contributing $\left(N_{\mathrm{wg}}^{\mathrm{ON}}=14\right)$ and 18 waveguides not contributing $\left(N_{\mathrm{wg}}^{\mathrm{OFF}}=18\right)$ to the output. The spectrum and the autocorrelation intensity profile of the input laser are shown as references. Similar spectra and temporal profiles were obtained at other output channels of the R-AWG. The output spectrum [Fig. 1(a)] of a single channel of a pulse shaper based on an R-AWG-DMD combination, with all the mirrors set to the ON-state, is a single peak. When grating waveguides of the R-AWG are sampled with $N_{\mathrm{wg}}^{\mathrm{ON}}=14$ and $N_{\mathrm{wg}}^{\mathrm{OFF}}=18$, additional transmission peaks are observed in the same channel output. These peaks are located symmetrically at wavelengths both lower and higher than the main peak, and are attributed to dynamic modifications of the FSR of the R-AWG [7]. The temporal response of a single channel of an R-AWG with all micromirrors set to the ON-state corresponds to a single broad output pulse with the pulsewidth about 13.5 times larger than that of the input laser [see Fig. 1(b)]. With a periodic structure of $N_{\mathrm{wg}}^{\mathrm{ON}}=14$ and $N_{\mathrm{wg}}^{\mathrm{OFF}}=18$ on the DMD, a pulse sequence 


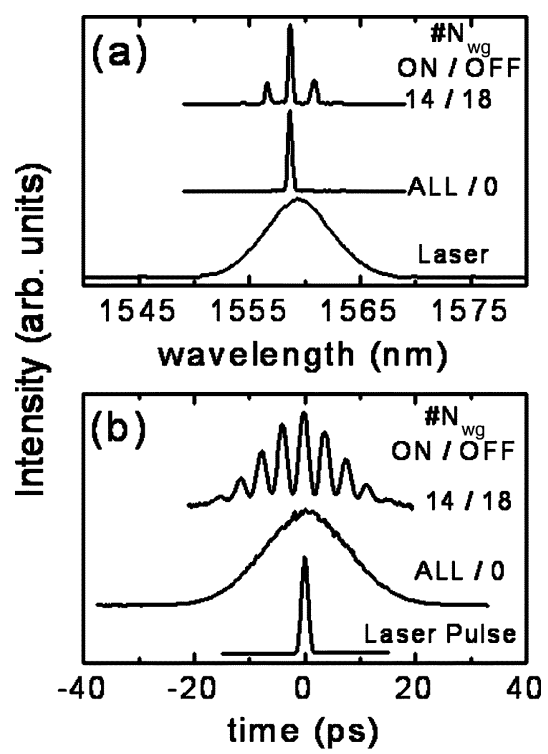

Fig. 1. (a) Output spectrum and (b) intensity autocorrelation measurements of a single output channel of an R-AWG with grating waveguides sampled with period $N_{\mathrm{wg}}^{\mathrm{ON}}=14, N_{\mathrm{wg}}^{\mathrm{OFF}}=18$ and with all mirrors of the DMD set to the ON-state. The spectrum and the intensity autocorrelation of the input laser source are also shown as references.

with pulse-to-pulse separation of $\sim 3.6 \mathrm{ps}$ was obtained in the same output channel of the R-AWG.

The performance of the R-AWG-DMD pulse-shaper [Fig. 1(b)] depends on the optical delay corresponding to the path-length increment $(\Delta L)$ between adjacent waveguides of the grating. In multiplexers designed for WDM applications $\Delta L$ is quite small, on the order of few micrometers [8]. This results in waveguide-to-waveguide delay time smaller than the temporal width of the input pulse. When a femtosecond laser is used as the input source for the multiplexer, low-intensity replicas of the input pulse are coupled into each waveguide of the grating. Each of these pulse replicas traverses a waveguide of the grating, is reflected at the external mirror, and leaves the waveguide delayed by the time corresponding to twice the length increment $\Delta L$.

When all the micromirrors of the DMD are set to ON-state, pulses returning from each grating waveguide overlap. This results in a single and broad output pulse at each output channel of the R-AWG, as observed in Fig. 1(b). However, when a periodic structure is created on the DMD, the pulsewidth becomes shorter than the effective propagation delay time between consecutive groups of waveguides, removing the temporal overlap between adjacent pulses. In the output pulse sequence, each period contributes a pulse, generating a burst of pulses. The repetition rate within the burst and the width of each pulse in the burst can be dynamically reconfigured by varying the periodic structure imposed on the DMD.

The output spectrum of a single channel of the pulse shaper is also modified by imposing a grating superstructure on the DMD. Dynamic control of the FSR in R-AWGs designed for WDM applications was demonstrated recently [7]. When the FSR is reduced, the spectrum of each output channel becomes multiply peaked. Since the spectrum and the output temporal

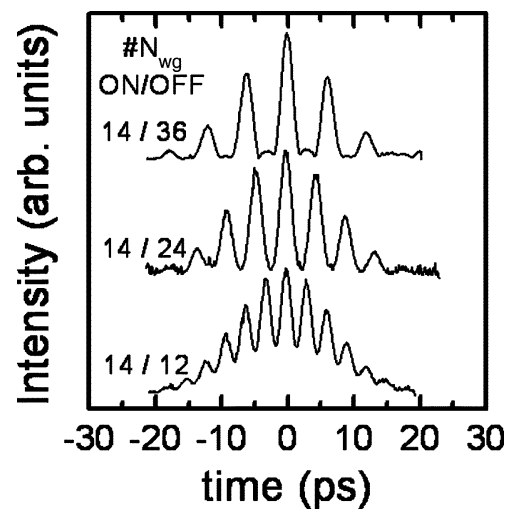

Fig. 2. Intensity autocorrelation measurements of a single output channel of an R-AWG with grating waveguides sampled with $N_{\mathrm{wg}}^{\mathrm{ON}}=14$ and different values of $N_{\mathrm{wg}}^{\mathrm{OFF}}$.

profile are related by a Fourier transform [7], multiply peaked spectra result in a train of pulses in the temporal domain, with the pulse repetition rate given by the inverse of the FSR [9], as clearly shown in Fig. 1.

In the active R-AWG-DMD approach, the pulse repetition rate can be modified by sampling the grating waveguides with different periods. Fig. 2 shows the temporal output response of a single output channel of an R-AWG with grating waveguides sampled with $N_{\mathrm{wg}}^{\mathrm{ON}}=14$ and different values of $N_{\mathrm{wg}}^{\mathrm{OFF}}$. When the sampling period $N_{\mathrm{wg}}^{\mathrm{ON}}+N_{\mathrm{wg}}^{\mathrm{OFF}}$ is increased from 26 to 50 , the pulse separation increased from 3.0 to $6.0 \mathrm{ps}$, respectively. In the data shown in Fig. 2, the widths of each pulse in all three output sequences are identical, since $N_{\mathrm{wg}}^{\mathrm{ON}}$ was kept constant. The number of pulses in the train also depends on the sampling period $N_{\mathrm{wg}}^{\mathrm{ON}}+N_{\mathrm{wg}}^{\mathrm{OFF}}$. A shorter sampling period resulted in a larger number of pulses in the train (see Fig. 2). However, the total temporal length and the envelope of the output pulse train are independent of the sampling period. R-AWGs were designed to have Gaussian passband output response [8]. Consequently, the electric field intensity distribution at the grating also has a Gaussian profile [10]. This results in a Gaussian envelope for the overall output pulse sequence (or a single pulse, when all the micromirrors are set to the ON-state). The total temporal length of the pulse sequence is related to the inverse of the AWG passband width [9]. R-AWGs used here were designed to have a $3-\mathrm{dB}$ bandwidth of $25 \mathrm{GHz}$. This results in a pulse train length of the order of 30 ps, as shown in Figs. 1 and 2.

In order to analyze the effect of the sampling period in the temporal output profile of the pulse shaper, we simulate the response of the device using a single Gaussian approximation [10]. Phase and intensity values are calculated for each waveguide of the grating. The output spectra are obtained by summing up the amplitudes, taking into account the corresponding phase. We have successfully used this approach to describe the electric field distribution in the grating of an R-AWG [10]. For the present case, this procedure is modified to account for the pulse response of the AWG. The phase information is converted into delay times for the calculation of time-dependent intensity for each output waveguide. Calculated intensity autocorrelation profiles of an R-AWG output channel with grating waveguides sampled with $N_{\mathrm{wg}}^{\mathrm{ON}}=14$ and different values of $N_{\mathrm{wg}}^{\mathrm{OFF}}$ are 

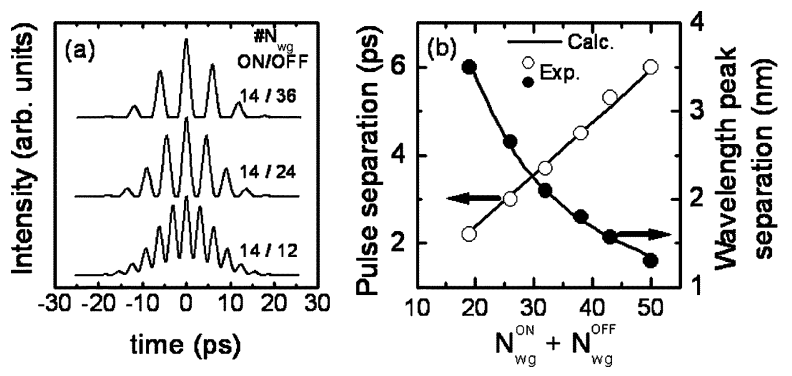

Fig. 3. Simulated intensity autocorrelation for a single output channel of an R-AWG with grating waveguides sampled with $N_{\mathrm{wg}}^{\mathrm{ON}}=14$ and different values of $N_{\mathrm{wF}}^{\mathrm{OFF}}$, and (b) comparison between calculated and measured pulse separation and peak wavelength as a function of the period $N_{\mathrm{wg}}^{\mathrm{ON}}+N_{\mathrm{wg}}^{\mathrm{OFF}}$.

shown in Fig. 3(a). Simulated temporal profiles are in excellent agreement with those obtained experimentally (see Fig. 2). When the waveguide grating of the R-AWG is sampled, the time separation between consecutive pulses in the burst $(\Delta \tau)$ and the peak wavelength separation $(\Delta \lambda)$ in the spectrum can be calculated using the expression for FSR [7], [9]

$$
\mathrm{FSR}=\Delta \tau^{-1}=\frac{c \Delta \lambda}{\lambda_{o}^{2}}=\frac{c}{2 n_{c}\left(N_{\mathrm{wg}}^{\mathrm{ON}}+N_{\mathrm{wg}}^{\mathrm{OFF}}\right) \Delta L}
$$

where, $c$ is the speed of light, $n_{c}$ is the refractive index of the grating waveguide, and $\lambda_{o}$ is the central channel wavelength. When $N_{\mathrm{wg}}^{\mathrm{ON}}=1$ and $N_{\mathrm{wg}}^{\mathrm{OFF}}=0$, (1) is reduced to the conventional definition of FSR, with the additional factor of two to account for the folded device design [7], [8]. Equation (1) shows that the time separation between pulses $\Delta \tau$ is directly proportional to the sampling period $N_{\mathrm{wg}}^{\mathrm{ON}}+N_{\mathrm{wg}}^{\mathrm{OFF}}$. In contrast, the wavelength separation $\Delta \lambda$ between the main peak and the adjacent spectral peaks is inversely proportional to $N_{\mathrm{wg}}^{\mathrm{ON}}+$ $N_{\text {wg }}^{\mathrm{OFF}}$. In order to verify the validity of our model, we show in Fig. 3(b) a comparison between calculated and measured $\Delta \tau$ and $\Delta \lambda$ for grating waveguides sampled with $N_{\mathrm{wg}}^{\mathrm{ON}}=14$ and different values of $N_{\mathrm{wg}}^{\mathrm{OFF}}$. The measured time separation $\Delta \tau$ increases linearly while the wavelength separation decreases inversely with the period $N_{\mathrm{wg}}^{\mathrm{ON}}+N_{\mathrm{wg}}^{\mathrm{OFF}}$, in excellent agreement with the values predicted by (1).

Using, the R-AWG-DMD approach, programmable sequences with pulses of different width can be obtained by periodically sampling the waveguides, at the surface terminating the grating, with different values of $N_{\mathrm{wg}}^{\mathrm{ON}}$. In each of the micromirror superstructure period, replicas of the input pulse traversing consecutive waveguide $\left(N_{\mathrm{wg}}^{\mathrm{ON}}\right)$ overlap. As a result, the width of each pulse in the train increases proportionally to $N_{\text {wg }}^{\mathrm{ON}}$. Fig. 4 shows examples of the temporal output response measured for an R-AWG with the waveguide grating sampled with different values of $N_{\mathrm{wg}}^{\mathrm{ON}}$. The $N_{\mathrm{wg}}^{\mathrm{OF}}$ was also varied in order to minimize overlap between consecutive pulses in the train. Fig. 4 shows that increased values of $N_{\mathrm{wg}}^{\mathrm{ON}}$ result in pulses with increased width. Intensity autocorrelation measurements shown in Fig. 4 demonstrate the pulsewidth increase of almost $40 \%$ with $N_{\mathrm{wg}}^{\mathrm{ON}}$ increased from 14 to 24 .

In summary, we demonstrate dynamic control of pulse sequence using a DST-PS that combines an R-AWG and an array of digital micromirrors. When all the mirrors of the DMD are set

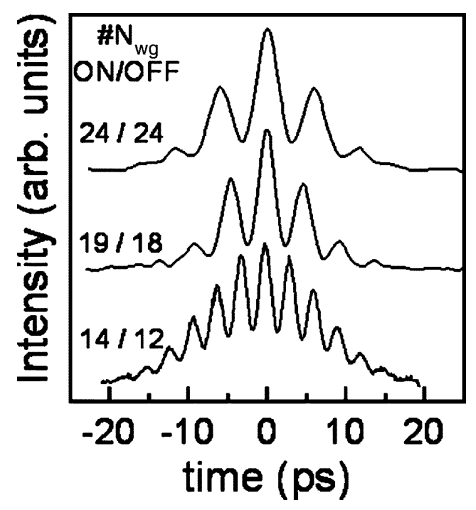

Fig. 4. Intensity autocorrelation measurements of a single output channel of an R-AWG with grating waveguides sampled with different values of $N_{\mathrm{wg}}^{\mathrm{ON}}$ and $N_{\mathrm{wg}}^{\mathrm{OFF}}$.

to the ON-state, a single broad pulse is observed at each output channel of the device. Sampling the waveguides of the grating with different periods resulted in output sequences with variable pulse separation within the burst. The width of the pulses in the burst can also be varied by changing the number of reflecting micromirrors in each period of the periodic structure formed on the DMD. Simulated temporal output response of the pulse-shaper is in excellent agreement with experiments.

\section{ACKNOWLEDGMENT}

The authors would like to thank Prof. R. Gale for advice on control and operation of DMDs.

\section{REFERENCES}

[1] H. P. Sardesai, C. C. Chang, and A. M. Weiner, "A femtosecond code-division multiple-access communication system test-bed," $J$. Lightw. Technol., vol. 16, no. 11, pp. 1953-1964, Nov. 1998.

[2] M. C. Hauer, J. E. McGeehan, S. Kumar, J. D. Touch, J. Bannister, E. R. Lyons, C. H. Lin, A. A. Au, H. P. Lee, D. S. Starodubov, and A. E. Willner, "Optically assisted internet routing using arrays of novel dynamically reconfigurable FBG-based correlators," J. Lightw. Technol., vol. 21, no. 11, pp. 2765-2776, Nov. 2003.

[3] A. Weiner, "Femtosecond pulse shaping using spatial light modulators," Rev. Sci. Instrum., vol. 71, pp. 1929-1960, 2000.

[4] M. R. Fetterman, D. Goswami, D. Keusters, W. Yang, J.-K. Rhee, and W. S. Warren, "Ultrafast pulse shaping: amplification and characterization," Opt. Express, vol. 3, pp. 366-375, 1998.

[5] D. E. Leaird and A. M. Weiner, "Femtosecond direct space-to-time pulse shaping," IEEE J. Quantum Electron., vol. 37, no. 4, pp. 494-504, Apr. 2001.

[6] D. E. Leaird, A. M. Weiner, S. Shen, A. Sugita, S. Kamei, M. Ishi, and K. Okamoto, "High repetition rate femtosecond WDM pulse generation using direct space-to-time pulse shapers and arrayed waveguide gratings," Opt. Quantum Electron., vol. 33, pp. 811-826, 2001.

[7] A. A. Bernussi, L. Grave de Peralta, M. Knapczyk, R. Gale, and H. Temkin, "Reconfigurable sampling of the electric field at the reflecting surface of folded arrayed waveguide grating multiplexers," IEEE Photon. Technol. Lett., vol. 16, no. 10, pp. 2257-2259, Oct. 2004.

[8] L. Grave de Peralta, A. A. Bernussi, S. Frisbie, R. Gale, and H. Temkin, "Reflective arrayed waveguide grating multiplexer," IEEE Photon. Technol. Lett., vol. 15, no. 10, pp. 1398-1400, Oct. 2003.

[9] D. E. Leaird, S. Shen, A. M. Weiner, A. Sigita, S. Kamei, M. Ishii, and K. Okamoto, "Generation of high-repetition-rate WDM pulse trains on arrayed-waveguide grating," IEEE Photon. Technol. Lett., vol. 13, no. 3, pp. 221-223, Mar. 2001.

[10] A. A. Bernussi, L. Grave de Peralta, and H. Temkin, "Electric field distribution in a grating of a folded arrayed-waveguide multiplexer," IEEE Photon. Technol. Lett., vol. 16, no. 2, pp. 488-490, Feb. 2004. 